\title{
A Proposal on the Marine Traffic Supporting System in VTS area
}

\author{
Hyong-Ki Lee* · Seong-Rok Chang** ·Gi-Nam Jeong*** • †Young-Soo Park \\ *, + Ship Operation Training Center, Korea Maritime University, Busan 606-791, Korea \\ ** Division of Safety Engineering, Pukyong National University, Busan 608-737, Korea \\ *** Busan Regional Maritime Affairs \& Port Office, Ministry of Land, Transport and Maritime Affairs
}

\begin{abstract}
In port and its approach channel, traffic accidents such as collision, aground, minor collision have reached about $77 \%$ of total marine casualty in the area. In this paper, an attempt to enhance the safe navigation was proposed by offering marine traffic supporting system which helps VTS operator assist vessel effectively with the quantitative assessment on difficulty of each vessel. The system collects navigation data from onboard AIS, assesses the data in assessment mode to analyze the navigation difficulties of each vessel and displays the degree of danger of each vessel on the ECDIS in real-time to decide the intervention time or order of priority for VTS operator. The effectiveness of the system was verified by the VTS operators in Korea.
\end{abstract}

Key words : Vessel Traffic Service(VTS), traffic accident, assessment model, Automatic Identification System(AIS), Electronic Chart Displaynand Information System(ECDIS)

\section{Introduction}

Vessels navigating in ports or along coast encounter with many other vessels and/or obstacles. Korea government has operated 15 vessel traffic service(VTS) centers for safety and efficiency of navigation and protection of the marine environment since 1993. Generally, ships entering a VTS area have to report to the authorities, receive traffic information or warning and may be tracked by the VTS control center. According to previous researches, VTS has contribute to protection of marine casualties and has been effective on traffic accidents such as collision, aground and minor collision.(Johnson, 1978; Kuroda et al, 1990; Canada Coast Guard, 1991)

In recent 10 years(1998-2007), the marine casualty had been occurred at the rate of $14.6 \%$ in port and its approach channel and $65.6 \%$ in the vicinity of coastal water in Korea. And, traffic accidents in VTS area had reached 76.7 \%.[KMST, 1998-2007] Based on above statistics, it is considered that VTS control system is be reinforced to reduce traffic accidents effectively in spite of the advantage of VTS,

So, this paper intends to improve VTS control procedure by operators's subjective experience and knowledge and provides marine traffic supporting system based on the quantitative assessment of the marine traffic environment for VTS operators. It will help VTS operators identify rapidly vessels faced on danger and decide the priority among many vessel under control.

The proposed system on this paper displays dangerousness of each vessel on the computer screen in real time by assessment model.

The effectiveness of the system was verified by the VTS operators in Korea VTS center.

\section{Assessment Model for Marine Traffic Supporting System}

It is necessary to adopt assessment model to evaluate and calculate dangerousness of each vessel quantitatively. The adopted model, which quantitatively expresses the degree of stress imposed by topographical and traffic environments on a mariner, is called the Environmental Stress $\operatorname{Model}(E S$ model ; Inoue, 2000).

The Environment Stress Model is specified as below.

\subsection{Environmental Conditions}

The elements of environmental conditions that can be taken into account in the model are as follows;

1) Topographical conditions such as land, shoals, shore protection, breakwaters, buoys, fishing nets, moored ships

\footnotetext{
† Corresponding author : youngsoo@hhu.ac.kr 051)410-5085

* hyongki@hhu.ac.kr 051)410-4201

** srchang@pknu.ac.kr 051)629-6468

*** safer@hanafos.com 051)620-5806
} 
and other fixed or floating obstacles

2) Traffic conditions such as the density of other ships and traffic flow

3) External disturbances such as winds and currents

\subsection{Model Structure}

In the most case of ocean-going navigation, there are no restrictions on the sea for maneuvering and there is sufficient TTC(Time to collision), regardless of the ship direction. So no stress is imposed on the mariner and he feels no difficulty in ship handling. But the area available for maneuvering in narrow waterways is restricted, and there is little TTC, regardless of the ships direction. Therefore, the topographical environment causes the mariner considerable stress and creates difficulty in ship handling. When other ships are present in the vicinity, and there is a danger of collision with other ships according to the direction of sailing, the mariner is taken under additional stress. This stress becomes particularly great when there is little TTC, regardless of the direction of the ship.

The ES model is composed of the following three parts. The conversion formula shown in equation is given by regression equations found through experiments of ship handling simulation with 31 subjects and questionnaire 573 answers(Inoue et al, 1998).

1) Evaluation of ship handling difficulty arising from restrictions on the water area available for maneuvering.

A quantitative index expressing the degree of stress forced on the mariner by topographical restrictions $\left(E S_{L}\right.$ value : $E S$ value for Land) is calculated on the basis of the TTC with any obstacles.

Based on this concept, the value $E S_{L}$ is calculated with the common procedures shown below;

(1) Consider the ship's course in the range of $180^{\circ}$.

(2) Calculate the TTC for each one degree in the range of $\pm 90^{\circ}$ centered on the present course.

(3) Convert the TTC into the mariners perception of safety on each one degree.

$S J_{L}$ (Subjective Judgement for Land) is subjective judgment of mariners in relation to TTC with obstacles. The scales of the subjective judgment consist of numeric values with seven steps from 0 (extremely safe) to 6 (extremely dangerous). $\alpha$ and $\beta$ is coefficients determined by the size of own ship.

$$
\begin{array}{ll}
S J_{L}=\alpha \times(R / V)+\beta & \\
\alpha=-0.00092 \times \log 10(G T)+0.0099 & \text { GT } \leq 10,000 \\
\alpha=-0.006671 \times \exp \{-7 \times 10-6(G T)\} & \text { GT }>10,000
\end{array}
$$

$\beta=-3.82$

where, $R$ : distance to topographical restriction $V:$ speed

(4) Sum the values of $S J_{L}$ within the range of course $\pm 90^{\circ}$ to find the stress values as follows;

$$
\begin{aligned}
& E S_{L}=\sum\left[S J_{L}\right]_{i} \\
& \text { Where, } i=-90^{\circ} \sim+90^{\circ}
\end{aligned}
$$

2) Evaluation of ship handling difficulty arising from restrictions on the freedom to make collision-avoidance manoeuvres.

A quantitative index expressing the degree of stress forced on the mariner by traffic congestion $\left(E S_{S}\right.$ value : $E S$ value for Ship) is calculated on the basis of the TTC with ships.

Based on this concept, the value $E S_{S}$ is calculated with the common procedures shown below;

(1) Consider the ship's course in the range of $180^{\circ}$.

(2) Calculate the TTC for each one degree in the range of $\pm 90^{\circ}$ centered on the present course.

(3) Convert the TTC into the mariners perception of safety on each one degree.

$S J_{S}($ Subjective Judgement for Ship) is subjective judgment of mariners in relation to TTC with ships. The scales of the subjective judgment consist of numeric values with seven steps from 0(extremely safe) to 6(extremely dangerous). $\alpha$ and $\beta$ is coefficients determined by the combination of the size and situation of/between own ship and target ship.

$$
\begin{aligned}
& S J_{S}=\alpha \times(T T C \times V / L m)+\beta \\
& \alpha=0.00192 \times \operatorname{Lm} \\
& \beta=-0.65 \times \ln (\mathrm{Lm})-2.07 \quad ; \text { Crossing from STBD } \\
& \beta=-0.65 \times \ln (\mathrm{Lm})-2.35: \text { Crossing from Port } \\
& \beta=-0.65 \times \ln (\mathrm{Lm})-2.07 \quad: \text { Head on situation } \\
& \beta=-0.65 \times \ln (\mathrm{Lm})-0.85: \text { Overtaken situation } \\
& \text { where, TTC : Time to Collision } \\
& \text { V : Relative speed } \\
& \text { Lm : Mean of two ship's length }
\end{aligned}
$$

(4) Sum the values of $S J_{S}$ within the range of course $\pm 90^{\circ}$ to find the stress values as follows;

$$
\begin{aligned}
& E S_{S}=\sum\left[S J_{S}\right]_{i} \\
& \text { Where, } i=-90^{\circ} \sim+90^{\circ}
\end{aligned}
$$

3) Aggregate evaluation of ship handling difficulty forced by both topographical and traffic environments, in which the stress value $\left(E S_{A}\right.$ value : $E S$ value for Aggregation) is 
derived by superimposing the value $E S_{L}$ and the value $E S_{S}$.

In the respective calculations of the values $E S_{L}$ and $E S_{S}$, a common index was used and the same algorithm was introduced to perform simultaneous aggregate evaluations of ship handling difficulty as experienced in encounters with other ships in ports and narrow waterways.

$$
E S_{A}=\sum_{\varphi=0}^{ \pm 90} \sum \max \left[S J_{L}, S J_{S}\right]
$$

\subsection{Classification of Stress Value}

If there is no danger in all directions, the $S J$ (Subjective Judgement) value of 0 extends over $180^{\circ}\left(-90^{\circ} \sim+90^{\circ}\right)$, so this becomes zero as the minimum stress value. If there is an immediate danger, regardless of the ship's direction, the $S J$ value of 6 extends over $180^{\circ}$, so $6 \times 180 \fallingdotseq 1000$ is assigned as the maximum stress value. The stress ranking is set up by classifying the range of stress values as 0 to 1000 , as shown in Table 1.

Table 1 Stress ranking and acceptance criteria

\begin{tabular}{|c|c|c|c|c|}
\hline \multicolumn{2}{|c|}{ SJ : Mariner's Judgement } & \multirow{2}{*}{$\begin{array}{c}\text { Evaluate } \\
\sum[S J]_{i} \\
-[0]-\end{array}$} & $\begin{array}{l}\text { Stress } \\
\text { Ranking }\end{array}$ & \multirow[t]{2}{*}{$\begin{array}{c}\text { Acceptance } \\
\text { Criteria }\end{array}$} \\
\hline 0 & Extremely safe & & \multirow{3}{*}{ Negligible } & \\
\hline 1 & Fairly safe & \multirow{6}{*}{$\begin{array}{r}-[500] \\
-[750] \\
-[900] \\
-[1000]\end{array}$} & & \multirow{3}{*}{ Acceptable } \\
\hline 2 & Somewhat safe & & & \\
\hline 3 & Neither safe or dangerous & & & \\
\hline 4 & Somewhat dangerous & & Ma & \multirow{3}{*}{ Unacceptable } \\
\hline 5 & Fairly dangerous & & Critical & \\
\hline b & Extremely dangerous & & Catastrophic & \\
\hline
\end{tabular}

The rank of stress can be classified according to the extent to which a dangerous situation causes a particular $S J$ value in the range of $\pm 90^{\circ}$ around the present ships course. In the model, a situation giving the same $S J$ value, regardless of direction, was taken as the standard situation. The relationship between each stress ranking and the acceptable level was found through ship handling simulator experiments and a questionnaire.

The ES model, therefore, allows us to judge how great a stress value will be when it is no longer acceptable and to point out the disadvantages of the topographical and traffic situation in ports and waterways.

\subsection{Calibration of Model Output}

To verify the outputs of $E S$ model, a calibration was attempted by using a ship handling simulator.(Inoue, 2000). In trials, several scenarios in which the ship encountered other ships in a curved or narrow waterway were performed. $E S_{A}$ values were calculated from the results of trials, and pulse of heartbeat of the mariner subjected to the simulation trial was measured at the same time. The correlation between the stress values derived from the ES model and the indices of physical stress obtained from the spectral analysis of the change of heartbeat is shown in Fig. 1. In this figure, the physical stress index means the number of heartbeat.

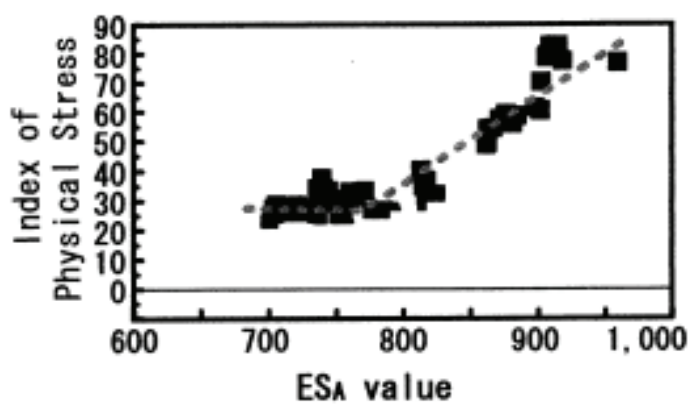

Fig. 1 Calibration of model output

It was demonstrated the validity of the model that the index of physical stress increases as the $E S_{A}$ value increases in the unacceptable area to a value of more than 750.

\section{Construction of Marine Traffic Supporting System}

The Marine Traffic Supporting System aims to display the degree of danger through calculation of stress value of each ship on computer screen for improving the marine traffic safety in the difficult waterways such as narrow channels, ports and any other restricted areas.

To achieve the purpose, it is necessary to adopt a quantitative assessment model to analyze the navigational difficulties of each ship in the VTS area. All factors to be needed in the assessment model is derived from data of Automatic Identification System(AIS) and Electronic Chart Displaynand Information System(ECDIS). After processing for $E S_{A}$, the system displays dangerousness of each ship on ECDIS in real time to help VTS operator's decision.

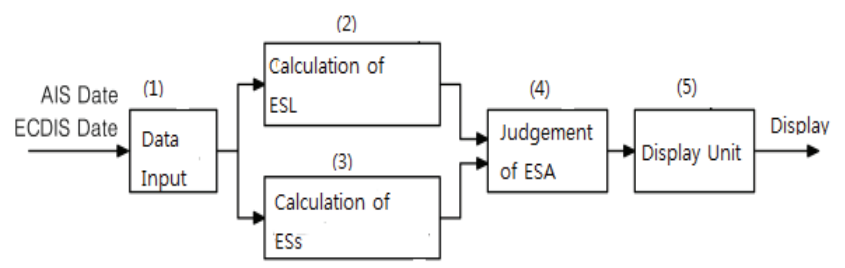

Fig. 2 Data process diagram of the system 
Fig.2 shows the all component of Marine Traffic Supporting System. Data Input unit(1) receives topographical information from ECDIS and ship's speed, course and size from AIS. Calculation of $E S_{L}$ unit(2) is calculating $E S_{L}$ value of each vessel based topographical information from ESDIS. Calculation of $E S_{S}$ unit(3) is calculating $E S_{S}$ value of each vessel based on data from AIS. These two parts(2, 3) are programmed to obtain subjective stress value using the formula for obstacles such land or another ships. Judgement of $E S_{A}$ unit(4) decides Environmental Stress for Aggregation as higher valve between $S J_{L}$ and $S J_{S}$ and then judges stress ranking comparing $E S_{A}$ with previously designating standard level. On this parer, standard level is designated as 4 steps, which are Negligible(0-499), Marginal(500-699), Critical(700-899), Catastrophic(9001,000). Display unit(5) shows danger of each ship based on $E S_{A}$ value and selected stress level. on the screen. Danger of each ship is displayed in different color such as brown(Negligible), blue(Marginal), yellow(Critical) and red(Catastrophic). And also, Display unit(5) displays and saves colored track of ships to make a proper counter measures to protect marine casualty in specific area.

Fig. 3 and 4 show the concept diagram and actual installation.

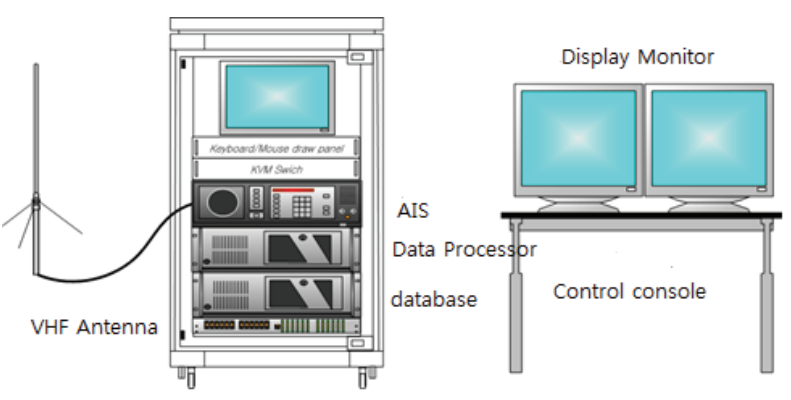

Fig. 3 Concept Diagram of system

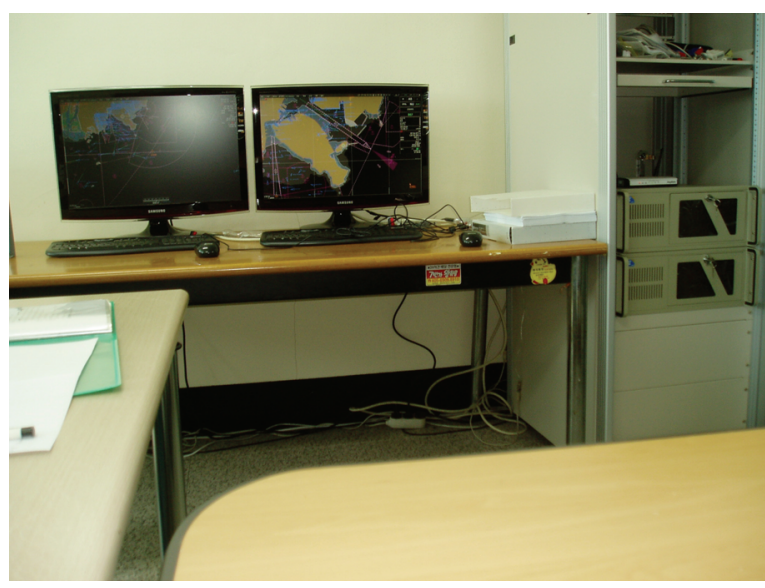

Fig. 4 Actual installation of system
Fig. 5 shows various danger rating of vessels in Busan port in real time. VTS operator can identify easily dangerous vessels and make the priority under control.

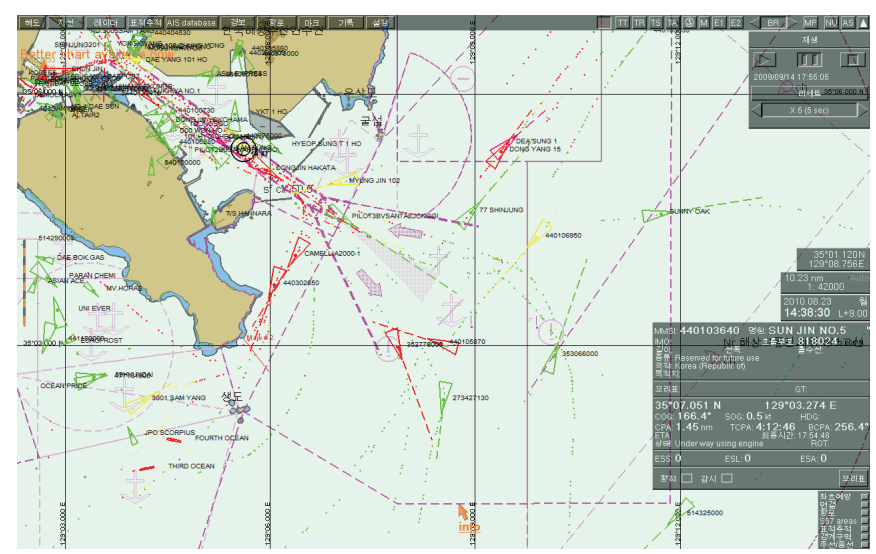

Fig. 5 Display of ship's danger

Fig. 6 shows ship's tracks in area between Ulsan and Busan.

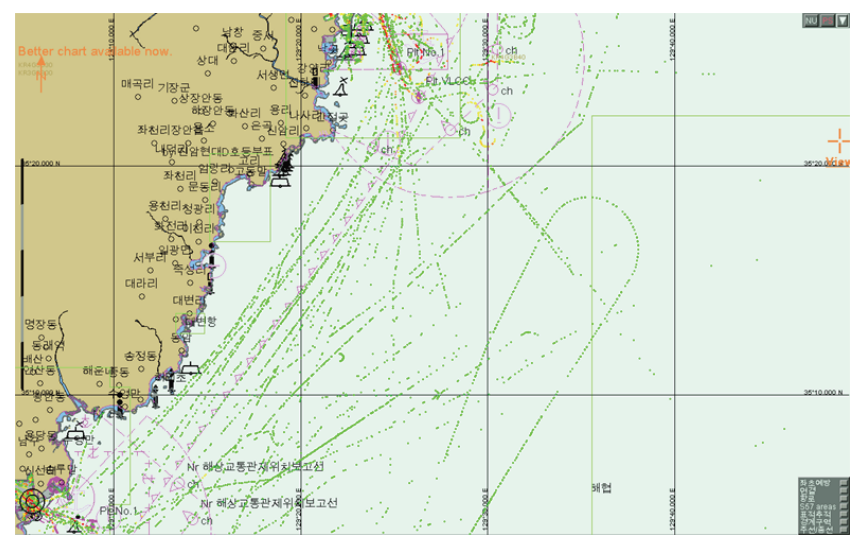

Fig. 6 Display of ship's track

It is likely to be natural that red track(Catastrophic) is displayed in vicinity of port entrance due to traffic congestion. But, the system shows that some area apart from the port also has red tracks, which means other countermeasure to be needed for safety of navigation.

\section{Verifying of Marine Traffic Supporting System}

The questionnaire targeting 22 VTS operators was conducted to verify the effectiveness of the Marine Traffic Supporting System.

In questionnaire, 4 different scenarios on the black and white(Fig.7) were served and asked to mark all vessels to be considered dangerous and to be controlled respectively. 


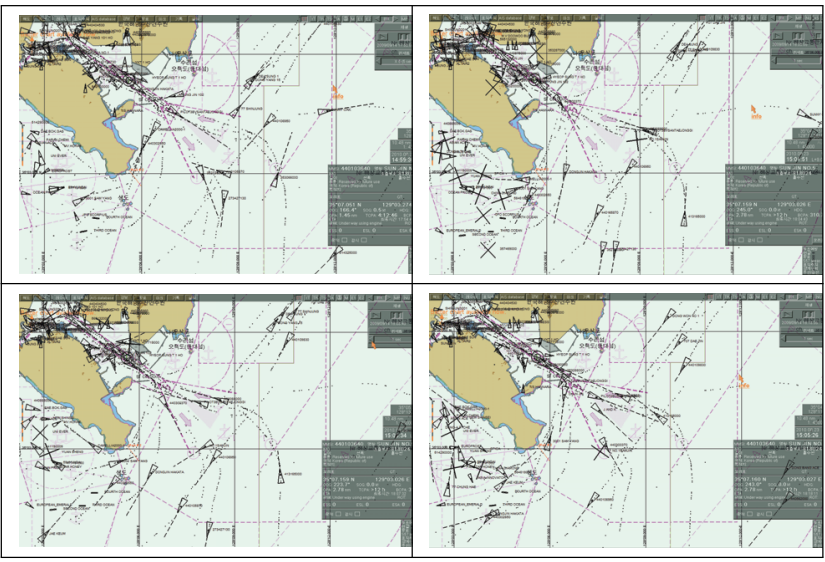

Fig. 74 Scenarios in questionnaire

Table 2 shows the results after comparing same scenarios colored on the system to identify difference of dangerousness between VTS operators and navigators. It means that VTS operators consider only $21.5 \%$ of dangerous vessels by ship's operators as dangerous vessel and 25.0\% as to be needed controlled promptly.

Table 2 Difference of dangerousness

\begin{tabular}{|l|c|c|c|}
\hline \multirow{2}{*}{} & \multirow{2}{*}{$\begin{array}{l}\text { On the } \\
\text { system }\end{array}$} & \multicolumn{2}{|c|}{ VTS Operator's response } \\
\cline { 3 - 4 } & & Dangerous & Controlled \\
\hline Catastrophic & 24 & $4.90 \pm 2.66$ & $5.50 \pm 3.61$ \\
\hline Critical & 6 & $1.55 \pm 1.26$ & $2.00 \pm 1.38$ \\
\hline Marginal & 30 & $2.91 \pm 2.89$ & $3.45 \pm 2.69$ \\
\hline
\end{tabular}

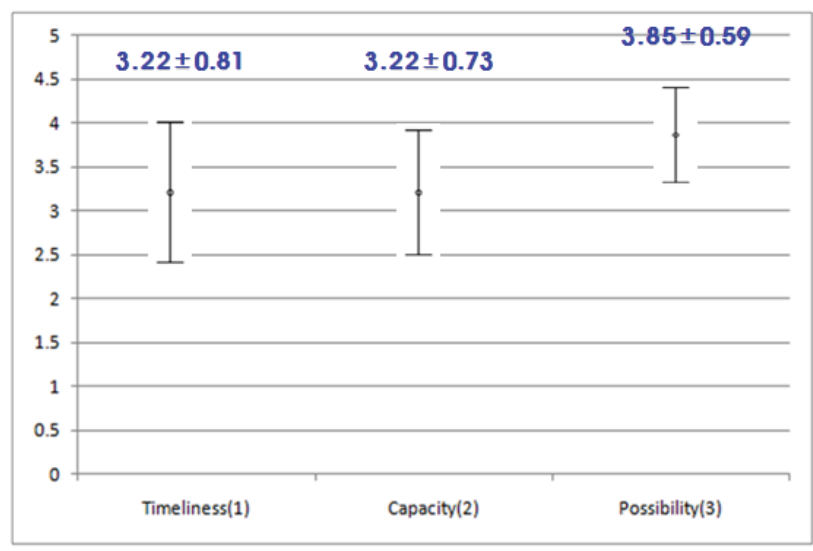

Fig. 8 Effectiveness of the system

Table 4 and Fig. 8 show the results to evaluate timeliness of danger rating of vessel on the system(1), capacity to help operator's decision on the point of time starting control(2) and possibility to reduce operator's human error on controlling vessel(3).
Table 3 Effectiveness of the system

\begin{tabular}{|l|c|}
\hline & Mean \pm S.D. \\
\hline Timeliness(1) & $3.22 \pm 0.81$ \\
\hline Capacity(2) & $3.22 \pm 0.73$ \\
\hline Possibility(3) & $3.85 \pm 0.59$ \\
\hline
\end{tabular}

VTS operators evaluated the marine traffic supporting system positive especially for possibility to reduce operator's human error on controlling vessel.

\section{Conclusion}

The maritime casualty has largely occurred in port and its approach channel and traffic accidents such as collision, aground, minor collision has reached about $77 \%$ of total marine casualty in the area. To eliminate the traffic accident in the area, we considered controlling the skill of a mariner, environment in vicinity of ship and the maneuvering performance etc.

This paper focuses on the improvement of traffic environment by providing VTS operator with marine traffic supporting system. It is estimated to help VTS operator assist vessel effectively with the quantitative assessment instead of a subjective judgement based on his/her own experience and knowledge.

The marine traffic supporting system is collecting navigation data from onboard AIS, assessing the data in assessment model $\left(E S_{A}\right.$ model $)$ to analyze the navigation difficulties of each vessel. And it displays the degree of danger of each vessel on the ECDIS in real time to identify rapidly vessels faced on danger and to decide the priority among many vessels under control.

The real-time system is supposed to eliminate effectively the human error of VTS operators and subsequently reduce the traffic accidents in the VTS area. The effectiveness of the system was verified by the VTS operators in Korea VTS center. VTS operators evaluated the system positive ,which indicate the danger rating of vessel(3.22 \pm 0.81$)$, the point of time starting control(3.22 \pm 0.73$)$ and possibility to reduce operator's human error on controlling vessel(3.85 \pm 0.59).

This marine traffic supporting system is expected to contribute to further wide range of applications in the safety, traffic control and training sector. 


\section{Reference}

[1] Canadian Coast Guard(1991). 1991 VTS Update Study. Otawa, Canada

[2] Inoue, K.(2000), "Evaluation Method of Ship handling Difficulty for Navigation in Restricted and Congested Waterways", The Journal of Navigation, The Royal Institute of Navigation, Vol.53, No.1, pp.167 180 .

[3] Inoue, K. and et al(1998), Modeling of Mariners' Perception of Safety, The Journal of Navigation, The Japan Institute of Navigation, No.98, pp.235 245, 1998

[4] Johnson D.R.(1978). Recent Trends in Navigation Safety in the Dover Strait. Proc. of 3rd International Symposium on Marine Traffic Service. Liverpool, UK

[5] Kuroda K \& Kita H(1990). Safety Assessment of Waterway Network in Bay Area. Proc. of 27th International Navigation Congress. Osaka, Japan

[6] Korea Maritime Safety Tribunal(1998-2007), Statistics of Marine casualty, http://www.kmst.go.kr

Received 11 November 2010

Revised 13 December 2010

Accepted 13 December 2010 